
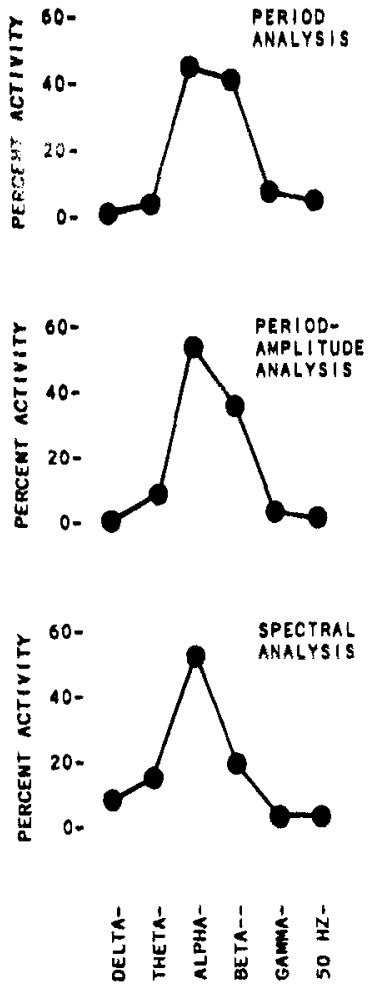

Fig. 2. Percent EEG activity in each of the standard EEG frequency bands over $2501.024-\mathrm{sec}$ data records by period, period-amplitude, and spectral analy ses.

procedures may be safely employed to obtain an ordering of intervals based on the relative prominence of activity within a particular frequency band, necessary in studies involving EEG conditioning.

Period analytic methods effectively estimate the percent power in the EEG bands of major interest. Percent alpha, as measured using standard spectral methods, correlates .79 with the period and .82 with the period-amplitude estimates of the same percentages. Similarly, the correlations for percent beta frequency activity are .78 and .81 . Thus, it appears that the simpler period analytic methods may be used to estimate the percentage of spectral power in the alpha and beta frequency bands of the waking human EEG.

\section{REFERENCES}

Beatty, J. Effects of initial alpha wave abundance and operant training procedures on occipital alpha and beta wave activity. Psychonomic Science, 1971, 23 197-199.

Beatty, J. Similar effects of feedback signals and instructional information on EEG activity. Physiology \& Behavior, 1972, 9, 151-154.

Beatty, J., \& Kornfeld, C. Relative independence of conditioned EEG changes and cardiac and respiratory activity. Physiology \& Behavior, 1972, 9, 733-736.

Carrie, J, R. G \& Frost, J. D., Jr. A small computer sy stem for EEG wavelength-amplitude profile analysis. Biomedical Computing, $1971,2,251-263$.

Cooley, J. W., \& Tukey, J. W. An algorithm for the machine calculation of complex Fourier series. Mathematics of Computation, 1965, 19, 297-301.

Jenkins, G. M., \& Watts, D. G. Spectral analysis and its applications. San Francisco: Holden Day, 1968.

Saltzberg, B. Period analysis. In M. Matousek (Ed.), Evaluation of bioelectrical data from brain, nerve and muscle, II. Part A. Frequency and correlation analysis. In A. Reymond (Ed.), Handbook of electroencephalography and clinical neurophysiology. 5 A. Amsterdam: Elsevier, 1973.

Siegel, S. Nonparametric statistics for the behavioral sciences. New York: McGraw-Hill, 1956.

Welch, P. D. The use of fast Fourier transform for the estimation of power spectra: A method based on time averaging over short-modified periodograms. IEEE Transactions on Audio Electroacoustics, AU-15, 1967, 70-73.

\title{
A minicomputer program for stimulus control and analog data for discrete trial paradigms in biological preparations: Classical conditioning*
}

\author{
ROBERT W. TAIT and I. GORMEZANO \\ University of lowa, Iowa City, lowa 52240
}

The application of computer technology to classical conditioning requires: control sof tware that will repeatedly generate stimulus events, and analog-to-digital (A/D) recording capabilities for the resolution of analog data from phasic response systems (e.g., nictitating membrane extension, leg flexion). An earlier software package implemented on a 4K PDP-8/E (Millenson, Kehoe, Tait, \& Gormezano, 1973) successfully met these requirements. However, the program's capabilities were restricted to the presentation of only two unique stimuli with a maximum of 4 sec duration, and data reduction in only uniphasic response systems. The present program, employing the same hardware, relaxed these previous constraints and extended the range of data analysis through the revamping of the interactive assembler, operating system, and on-line data processing routines.

*The creation of the program and the preparation of this report were supported by NSF Grant GB-41531.
The E's operations for classical conditioning can be conceptualized as involving two types of temporal 
intervals. One type of interval, the intertrial interval, is generally long and variable in duration, and during this interval the stimulus conditions are relatively constant. The second type of interval, the observation interval, is generally shorter and fixed in duration. Nested within the observation interval is a pattern of stimulation constituting a classical conditioning trial. Accordingly, it is during the observation interval that the $\mathrm{E}$ examines analog signals for responses made by the S. Generally, the analog signals from both uniphasic and multiphasic response systems are analyzed from permanent (ink written) oscillograph records, as, for example, from those reproduced in Figs. 1 and 2.

The response depicted in Fig. 1 represents the uniphasic rabbit's nictitating membrane response (cf. Gormezano, Schneiderman, Deaux, \& Fuentes, 1962), while the responses shown in Fig. 2 are examples of the multiphasic (sinusoidal) rabbit's jaw movement response (cf. Smith, DiLollo, \& Gormezano, 1966). In both figures, the illustrated pattern of stimulation consists of an auditory CS paired with a UCS (paraorbital shock for the nictitating membrane preparation and intraoral water delivery for the jaw-movement preparation). From these oscillograph records, a conditioned response (CR) is identified by an upward deflection in the response line of more than $1 \mathrm{~mm}$ from baseline (corresponding to $1 / 2 \mathrm{~mm}$ of extension) in the interval between S1(CS) onset and S2(UCS) onset. While changes in CR frequency and $\mathrm{CR}$ onset latency (time from S1 onset to the initiation of the upward deflection in the response record, cf. "1" in Fig. 1) have been routinely recovered from such records, the hand-scored measurement of other dependent variable measures becomes a very tedious, errorful, and time-consuming task. And yet, there is a need to be able to specify precisely the changes in CR topography in order to assess a number of increasingly sophisticated theoretical accounts of classical conditioning. Among such topographical dependent variable measures are those depicted by the numbers and letters in Fig. 1: (1) peak latency (No. 3), peak amplitude (No. 5), area to peak $(A+B+C)$, and total area $(\mathrm{A}+\mathrm{B}+\mathrm{C}+\mathrm{D})$ of the response in the interval from $\mathrm{S} 1$ onset to the end of the observation interval; and (2) maximum amplitude (No.4), latency of the maximum amplitude (No. 2), and the area (A+B) of the response in the S1-S2 interval. The present software package implements the routine collection of these measures. in addition, the program, by more efficiently utilizing the available hardware configuration, provides for more versatile experimental manipulations than one previously employed (Millenson, Kehoe, Tait, \& Gormezano, 1973).

\section{HARDWARE CONFIGURATION}

The computer configuration consists of a PDP-8/E with a 4K memory, an ASR-33 Teletype, 16 channels of A/D (AD 8/E) multiplexed by an AM8-EA controller, a DK8-EC real-time clock, two DR8-EA 12-bit digital input-output (I/O) buffers, and a high-speed paper-tape reader/punch. In the present system, the DR8-EA outputs are extended to a patch panel. The capability of changing the connections from the patch panel to the relay drivers of the stimulus devices yields maximum flexibility in the use of the digital outputs. The relay drivers control the delivery of stimuli to each of the six, sound-attenuated $S$ stations located in an adjoining room. Within each experimental chamber, there is a stimulus panel on which there are mounted two houselights, a speaker (for auditory CSs), and connectors for the delivery of shock (e.g., UCS for the nictitating membrane response) and water (e.g., UCS for the ja:w-movement response). Since a classical conditioning trial can be presented simultaneously to all S stations, the current program assumes this mode of stimulus delivery, and consequently, only one output bit of a DR8-EA is required to specify the occurrence of a stimulus to all chambers. (Future modifications of the software package will separate $\mathrm{S}$ stations for the independent delivery of stimuli). Furthermore, each of the experimental chambers has two analog input channels to the $\mathrm{A} / \mathrm{D}$, enabling the concomitant measurement of analog signals from two independent response systems for each S. Accordingly, 12 of the 16 available A/D channels are utilized. Each of the 12 analog inputs passes through a variable potentiometer which serves to (1) restrict the size of the voltage input to the sensitivity range $(1 \mathrm{~V} \mathrm{dc})$ of the $A / D$, and (2) permit adjustment for possible drift in the analog response baseline.

\section{GENERAL DESIGN}

An earlier software program designed to control stimuli and analyze analog responses in classical conditioning (Millenson, Kehoe, Tait, \& Gormezano, 1973) consisted of an assembler and operating system.

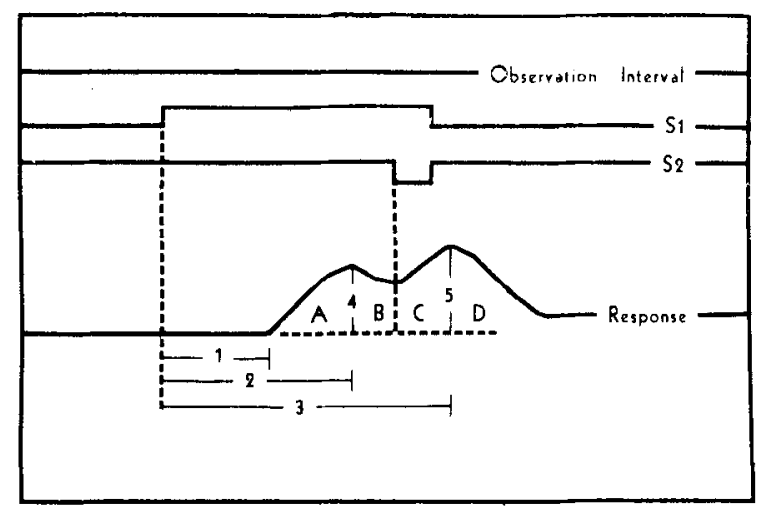

Fig. 1. A representation of a classical conditioning trial with S1 and S2 corresponding to the CS and UCS. The illustrated response is a nictitating membrane $C R$. The numbers and letters on the response represent different dependent variable measures. 


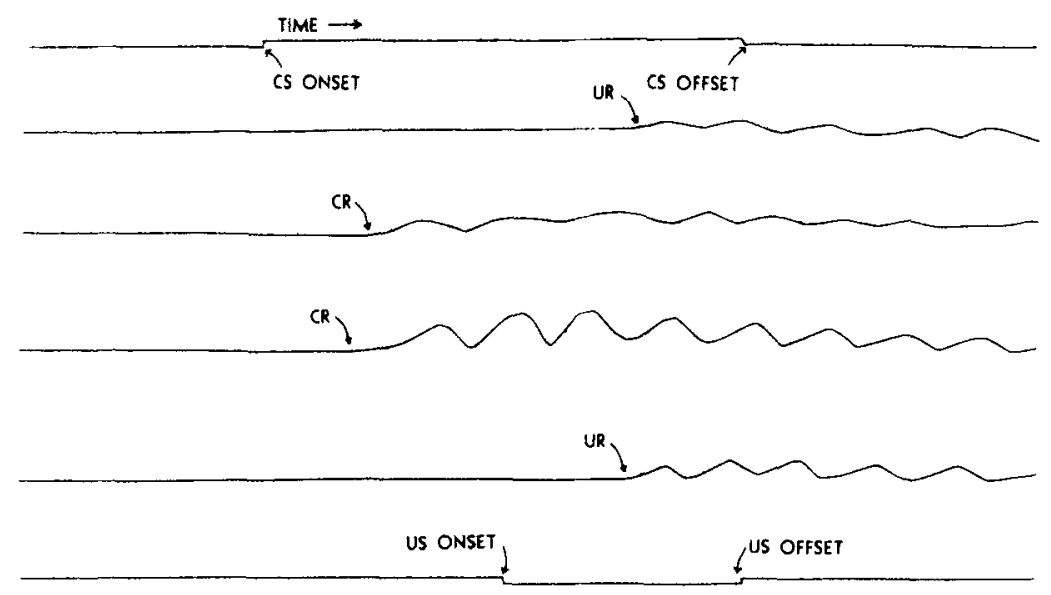

Fig. 2. A sample of oscillograph records for the jaw-movement responses of four $\mathrm{Ss}$ taken at a paper speed of $200 \mathrm{~mm} / \mathrm{sec}$.

The assembler encoded, from the Teletype, the stimulus parameters to be used by the operating system, which subsequently legislated the occurrence of stimulus events and data processing. While the program proved successful in the implementation of a variety of experimental manipulations for the conditioning of the rabbit's nictitating membrane response, several limitations became apparent. It was found that: (1) the conjunctive effects of the assembler design and memory core restriction limited the number of $\mathrm{A} / \mathrm{D}$ inputs to six, resulting in an underutilization of the available $A / D$ channels; (2) the stimuli and observation interval were restricted to durations of less than $4,095 \mathrm{msec}$; (3) a maximum of only three stimuli could be presented, and the duration of just two stimuli uniquely specifiable; (4) the A/D storage buffer was small and fixed in size, and as a result, the maximum $\mathrm{A} / \mathrm{D}$ sampling rate was an inverse function of observation interval; and (5) analog response analyses were limited to a few dependent variable measures, and were restricted to uniphasic response signals.

The present program alleviates the limitations of the previous program through the redesign and expansion of the capabilities of both the assembler and the operating system. The new assembler has the following modifications: (1) the core size of the assembler was reduced by employing only one mode of encoding trial-by-trial parameters; (2) the number of $A / D$ inputs was increased to $12 ;(3)$ a larger and now-adjustable storage buffer for each A/D channel was implemented; (4) the upper limit on the duration of the observation interval and stimulus events was increased by a factor of 10 by programming all stimuli in $10-\mathrm{msec}$ rather than 1-msec units; (5) the maximum number of different observation intervals occurring within an experimental session was extended from one to four; and (6) the upper limit on the number of uniquely programmable stimuli was increased from two to eight. The operating system was changed to utilize the expanded capabilities of the new assembler, and, in addition, it was provided with an enlarged analytic package which: (1) increased the number of dependent variable measures of the response, (2) extended analysis to include multiphasic response signals, and (3) incorporated an on-line dump of data to paper tape.

\section{THE ASSEMBLER}

The interactive assembler establishes the experimental design of a conditioning session by generating a Teletype conversation with the operator and by reading a parameter tape input. The Teletype conversation determines how many A/D inputs will be used, the A/D sampling rate, and the temporal parameters of the observation intervals and stimulus events. The ASCII-formatted parameter tape, punched off-line on the Teletype prior to the experimental session, programs the number of trials per session, intertrial intervals, and the pattern of stimuli on each trial. The properties of the assembler can best be illustrated by examining a representative sample of a Teletype preamble and a parameter tape.

\section{TELETYPE PREAMBLE}

Figure 3 presents a sample conversation with the assembler. The statements and questions of the assembler are underlined, with the remaining portions indicating the operator's replies. The initial Teletype message reminds the operator that stimulus input times are in 10-msec units. A request, "NUM A/D =," which demands the number of A/D inputs, follows. Values from 1 to 12 will advance the assembler to the next query, whereas an answer beyond this range results in a reiteration of the question.

The question "A/D STORED" required either a "1" or " $\phi$ " in reply. A " 1 " indicates that during the conditioning session, at least one trial will occur on which the operator will desire the storage of the complete point-by-point response topography until the end of the session, when the $A / D$ values are punched onto paper tape. If more than one such trial occurs, 
response topographies are carried as an average. A " $\phi$ " informs the assembler that this $A / D$ averaging and storing will not be used during the experimental session. If the reply is " 1 ," the A/D storage buffer is divided into half, one part reserved for the values that are stored until the end of the session and the other half used on each trial. At this point, the assembler calculates the maximum number of $A / D$ readings that can be stored for each $A / D$ channel and prints the decimal equivalent after the statement "BUFFER SIZE =."

The query "RESOL $=$ " asks for the $A / D$ resolution time. This is a demand for the desired interval, in milliseconds, between successive samplings of the A/D inputs. The $500-\mathrm{Hz}$ clock restricts the reply to multiples of two.

The query "RESP CRIT =" asks for the minimum change in the A/D record that will designate that a response has occurred. For example, with a $1 / 2-\mathrm{mm}$ extension of the nictitating membrane as the criterion used to identify a response, the current transducer configuration produces a change of $27_{8}$ in the A/D. The reply of $23_{10}$ found in Fig. 2 is the decimal equivalent of the $A / D$ response criterion.

The query "NUM OF OBS INT $=$ " requests the numbers of different observation intervals that will occur during the experimental session. Up to four different durations are accepted. The next question is "OBS:," which requires in reply a serial listing of the duration, in $10-\mathrm{msec}$ units, of each of the desired observation intervals. Subsequent to the reply, the assembler divides BUFFER SIZE by the requested observation intervals to obtain the corresponding minimum resolution value that will fill the $A / D$ storage buffer. These values, one for each observation interval, are then communicated to the operator by the statement "MIN RESOL ALLOWED =" followed by the printout, for example "0003, 0045." If the minimum resolution is shorter than the operator's desired resolution (as requested in reply to RESOL), the desired resolution will be employed. However, if the minimum value is longer than the desired resolution, then the minimum value will be employed. The resolution value that is operative for each interval is then employed to sample the $A / D$.

The question "NUM OF STIM =" asks for the number of different stimulus events that are desired during the experimental session. From 1 to 8 stimuli can be specified. A reply which requests values beyond this range produces a reiteration of the query. The reply (" 3 " in the example given in Fig. 3) is then used as a counter in a routine which first types the stimulus number "S1:" and then demands, with reference to the onset of the observation interval, the onset and offset times for that stimulus in $10-\mathrm{msec}$ units. If, during the listing of stimuli (e.g., "S1:," "S2:," and "S3:"), an offset time is less than the corresponding onset time, the assembler reinitializes the request "NUM OF STIM =." Since only one DR8-EA output bit is required per stimulus, the assembler assigns the stimulus number
ALL STIMULUS TIMES $=10 \mathrm{MSEC}$ UNITS

$\underline{N U M} \underline{A / D}=7$

A/D STORED? 1

BUFFER SIZE $=0309$

$\underline{\mathrm{RESOL}}=20$

$\underline{\text { RFSP CRIT }}=23$

NIM OF OBS INT $=2$

OBS: 150,500

MIN RESOL ALLOWED: 0003,0045

NUM OF STIM $=3$

S1: 20,70

s2: $\quad 50,70$

S3: $20,150: 1$

NUM OF TRIALS ON TAPE $=3$

RFADY

\begin{tabular}{|c|c|c|c|c|c|c|c|}
\hline CHANNEL & 1 & 2 & 3 & 4 & 5 & 6 & 7 \\
\hline$\pm \quad-3$ & 0000 & 0000 & 0002 & 0000 & 0001 & 0000 & 0000 \\
\hline \# CR & 0002 & 0001 & 0004 & 0006 & 0000 & 0004 & 0005 \\
\hline
\end{tabular}

Fig. 3. An example of a session preamble conversation with the assembler (upper portion) and the summary output from the operating system (lower portion). In the preamble, the assembler's underlined queries are followed by the user's replies.

(e.g., 1 for S1) to the corresponding DR8-EA output bit. If the operator wishes to change the output code, a colon is typed after the offset time and a new output code from the range of 1 to 8 is entered (e.g., "S3: 20, 150: 1"). Added versatility is gained through the ability to specify the output code for the stimuli. If two or more durations are required for the same stimulus, the time parameters can be assigned to separate stimuli and given the same output code. The trial parameters then determine which of the durations to employ. If the colon is not entered, then the normal output is assumed.

The final query "NUM TRIALS ON TAPE =" asks for the number of trials that have been encoded on the parameter tape. A carriage return after the reply sends the assembler into the parameter tape routine and shifts the mode of communication from the Teletype to the high-speed reader. The reply is used during the reading of the parameter tape as a checksum for the number of trials found on the tape.

\section{PARAMETER TAPE}

The accurate reading of the parameter tape requires the initial entry to be the specification of the number of times the operating system is to repeat the subsequent portion of the tape. The remaining entries on the tape contain a serial listing of two numbers per trial. The first number specifies, in seconds, the duration of the intertrial interval, while the second number is a numeric 
code specifying for that trial: whether or not storage of response topography is to occur; the observation interval; and stimulus. events. Each entry on the tape must be separated by either a "comma," "carriage return," or "linefeed." The last entry on the tape must be followed by a "period," which signals the end of the tape.

The assembler reads into memory the trial-by-trial listing from the parameter tape. The tape listing is restricted to one page of core, and since two consecutive 12-bit memory registers are employed to encode each trial, the upper limit of unique trial specifications is set at 64 . But, a sequence of unique trial specifications can be repeated 4,095 times and, consequently, the parameter tape can provide an exceedingly large number and variety of conditioning trials. However, the PDP-8/E's 12-bit registers impose an upper limit of $4,095 \mathrm{sec}$ on the duration of the intertrial interval, but they place no restriction on the $2^{11}$ possible combinations in the numeric code (technically $2^{11}-1$, since a parameter list register containing all zero bits is regarded as illegal by the operating system) for response topography storage $\left(2^{1}\right)$, observation interval $\left(2^{2}\right)$, and stimulus events $\left(2^{8}\right)$.

The bit representation that is used to generate the numeric code is presented in the register displayed in the upper portion of Fig. 4. Each box in the figure corresponds to 1 bit of a memory register. Starting with the extreme right-hand box, the first 8 consecutive boxes specify the possible stimuli, the 9 th box designates possible response topography collection, the 10 th box is not used, and the 11 th and 12th boxes specify the possible observation intervals. Below the 11 th and 12 th boxes are the binary bit designations of the four possible observation intervals. Above each box is a corresponding octal bit designation. A summation of the octal bit designation of the events that are desired on a particular trial constitutes the numeric code that is punched on the parameter tape.

An example of a parameter tape is presented in the lower portion of Fig. 4. The tape sequence begins with the entry " 2 " and indicates that the subsequent portion of the tape is to be repeated twice. The second entry, " 90 ," indicates that the first trial is to occur $90 \mathrm{sec}$ after the operating system is initiated, and the entry " 3 " is the numeric code that programs the occurrence of stimuli "S1" and "S2" on the first trial. The next two entries indicate that the second trial will occur $90 \mathrm{sec}$ after the first and will have a different stimulus configuration. The numeric code for the second trial, "2404," programs the second observation interval, response topography collection, and presentation of Stimulus "S3." The third trial is similar to the first, having a 90-sec intertrial interval and the presentation of "S1" and "S2." Thus, the illustrative tape sequence specifies an experimental session which contains six equally spaced trials, with the second and fifth trials requiring the averaging of response topographies.

During the reading in of the tape, the assembler

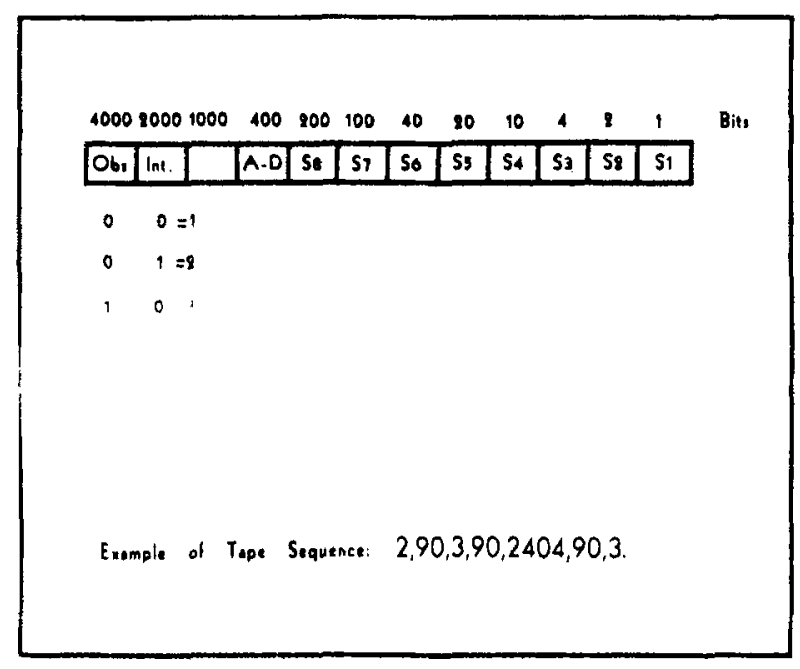

Fig. 4. The 12 boxes in the upper portion of the figure represent the memory register bit assignments for the observation interval (Obs Int.), special data processing (A-D), and stimulus events (S8 through S1). The octal bit designation above each box is used in the numeric code of a parameter tape to specify the occurrence of the corresponding event. Below the "Obs Int." boxes is the binary code employed to represent each of four observation intervals. The lower portion of the figure presents an example of a parameter tape sequence.

checks for several possible format errors. If an error is found, the assembler returns to the Teletype mode and prints the message "ERROR ON TAPE =" followed by " $R$," " $P$," " $F$," or "M." The " $R$ " error is signaled if the first entry on the tape is not a positive integer. A type "P" error indicates that a nonnumeric character has appeared in the numeric code. A type " $F$ " or " $M$ " error will be printed if there were fewer or more trials on the tape, respectively, than the number specified in the reply to the query "NUM TRIALS ON TAPE $=$. " After communicating the error, the assembler halts. Two options are then available to the operator. The error can be corrected and subsequently the tape reread by setting the rightmost bit of the switch register of the PDP-8/E console and then pushing the "CONT" switch. Alternatively, the error can be ignored and the assembler advanced to the "READY" message by setting the leftmost bit of the switch register and then pushing the "CONT" switch. If no errors are found on the tape, the assembler returns to the Teletype mode and types the message "READY." A carriage return will send the program into the operating system.

\section{THE OPERATING SYSTEM}

The operating system consists of a two-device interrupt skip chain combined with background data analyses and output. The devices causing interrupts are the Teletype keyboard and the $500-\mathrm{Hz}$ clock. The typing of the "CTRL" and " $Z$ " characters initiates the Teletype interrupt service routine and allows the operator to suspend the program and display on the PDP-8/E 
console the current readings from the analog input channel selected by the setting on the switch register. This suspend facility permits the adjustment of A/D balancing voltages at any time during the session to compensate for drifting response baselines. Setting the leftmost bit of the switch register resumes the experiment at the point where it was suspended. The $500-\mathrm{Hz}$ crystal clock interrupt service routine drives three software clocks. The first clock emits "pulses" at the rate of $1 / \mathrm{sec}$ and is used to time intertrial intervals. Upon the completion of the timing of an intertrial interval, the first clock is suspended and the other two clocks are activated as the trial begins. The second clock emits "pulses" every $10 \mathrm{msec}$ and is used to time the duration of the observation interval and stimulus events specified in the numeric code for this particular trial. The time base for the third clock depends on the A/D sampling rate. In turn, the sampling rate is determined by the resolution value associated with the observation interval employed on this trial.

Background analyses on the entries in the A/D buffer of each $A / D$ channel are conducted between each trial by a set of software routines. These routines first determine the number of $A / D$ readings which were taken between the onset (ON) and offset (OFF) of the observation interval (ON-OFF interval) and also between the onset of (1) the observation interval and an initial stimulus (ON-S1 interval), and (2) the first and second stimulus (S1-S2 interval). These latter two intervals are then employed by the software routines as reference points in the determination of the occurrence and temporal placement of a response. The following information is extracted from each $A / D$ buffer: (1) a determination of whether or not a response occurred in the ON-S1 interval; (2) the onset latency, peak latency, peak amplitude, area to the peak, and total area of a response in the S1-OFF interval; and (3) the maximum amplitude, latency of the maximum amplitude, and area of a response in the S1-S2 interval. After all A/D buffers have been examined, the data is punched in packed binary format onto paper tape. In addition, if the 400 bit of the numeric code for the previous trial was set, then each $A / D$ buffer is averaged with previous sets of readings to obtain an average response topography for each A/D channel. And finally, a running tabulation of the number of responses which occur in the ON-S1 interval (labelled -3 ) and in the S1-S2 interval (labelled CR) is maintained. With the completion of the last trial, the operating system prints on the Teletype the total number of trials in the experimental session and a summary table of the number of -3 responses and CRs per A/D channel. An example of the operating system summary output can be found in the lower portion of Fig. 3.

The classical conditioning software package described above is a program still under development. While this program has expanded the capabilities of an operating system for the classical conditioning situation and extended its applicability to any discrete multiphasic response system, several problems remain to be resolved. Specifically, the program is being modified to incorporate continuous response measurement, such as required in heart-rate conditioning, and to include response-contingent experimental designs.

\section{REFERENCES}

Gormezano, I., Schneiderman, N., Deaux, E., \& Fuentes, I. Nictitating membrane: Classical conditioning and extinction in the alb ino rabbit. Science, $1962,138,33-34$.

Millenson, J. R., Kehoe, E. J., Tait, R. W., \& Gormezano, I. A minicomputer program for control and data acquisition in classical conditioning. Behavior Research Methods \& Instrumentation, 1973, 5, 212-217.

Smith, M. C.. Dilollo, V., \& Gormezano, I. Conditioned jaw movement in the rabbit. Journal of Comparative \& Physiological Psychology, 1966;62, 479-483. 